\title{
The Effect of Traction Position in Cervical Traction Therapy Based on Dynamic Simulation Models
}

\author{
Lawrence K. F. Wong1 ${ }^{1}$ Zhiwei Luo¹, Nobuyuki Kurusu ${ }^{2}$ \\ ${ }^{1}$ Graduate School of System Informatics, Kobe University, Kobe, Japan \\ ${ }^{2}$ Kobe Factory of Minato Medical Science Co., LTD., Kobe, Japan \\ Email: wonglawrence@cs11.cs.kobe-u.ac.jp
}

How to cite this paper: Wong, L.K.F., Luo, Z.W. and Kurusu, N. (2017) The Effect of Traction Position in Cervical Traction Therapy Based on Dynamic Simulation Models. J. Biomedical Science and Engineering, 10, 243-256.

https://doi.org/10.4236/jbise.2017.105019

Received: February 9, 2017

Accepted: May 21, 2017

Published: May 24, 2017

Copyright $\odot 2017$ by authors and Scientific Research Publishing Inc. This work is licensed under the Creative Commons Attribution International License (CC BY 4.0).

http://creativecommons.org/licenses/by/4.0/

\begin{abstract}
This study describes the development of a cervical traction therapy simulation model that evaluates two types of the traction positions, namely the sitting position and the inclined position. An anatomically correct human skeleton model and two mechanical traction device models were constructed in simulations using a physics engine. The anterior and posterior intervertebral separations were measured at both positions with a series of traction forces $(60 \mathrm{~N}$ to $200 \mathrm{~N})$ and traction angles $\left(10^{\circ}\right.$ to $\left.40^{\circ}\right)$. The result suggested that the sitting position caused the subject to lean forward and as a result led to excessive anterior compression when traction angle is over 20 degrees. The inclined position creates greater intervertebral separations on both the anterior and posterior sides than the sitting position. This suggests that the inclined position may be more effective in increasing intervertebral separation than the sitting position.
\end{abstract}

\section{Keywords}

Cervical Traction Therapy, Spine, Traction Position, Dynamic Simulation

\section{Introduction}

Neck pain is a very common problem in the general population. In the "The Empowerment of People with Neck Pain: Introduction", Haldeman et al. [1] states that "most people can expect to experience some degree of neck pain in their lifetime". According to Judovich et al. [2], neck pain accounts for $15 \%$ of all soft tissue problems and $26 \%$ to $71 \%$ of the adult population experienced an episode of neck pain or stiffness in their lifetime. In an extensive literature review "The Burden and Determinants of Neck Pain in the General Population" [3], it 
was suggested that about $30 \%-50 \%$ of the general adult population experience neck pain that lasts for 12 months. There are many options that can help ease the pain on the neck. Cervical traction therapy, also known as non-surgical spinal decompression therapy, is one of the possible treatment options frequently included in rehabilitation programs.

\subsection{Cervical Traction Therapy}

Cervical traction therapy refers to any medical procedure that applies force along the inferior-superior axis of the spine to extend the cervical spine vertebrae. Its purpose is often to straighten the back, to relieve pressure on the spine and to increase blood flow to the injured area. For decades, traction therapy has been widely employed in nonsurgical therapies and rehabilitation to treat chronic neck pain caused by herniated discs and other injuries at the cervical spine region.

Cervical traction therapy can be mainly divided into manual traction and mechanical traction. A manual therapy is performed by a trained physical therapist or chiropractor with the patient usually lies flat in bed. In a mechanical traction therapy, the patient's head is attached to a head halter and is gently pulled by a motorized traction machine at a specific degree. Traction position varies in mechanical traction. In this case, the patient can sit upright on a chair at $90^{\circ}$, referred to as the sitting position, or at an inclined angle, known as the inclined position.

Cervical traction therapy has been widely adopted in clinics and rehabilitation centers. Over the year, many studies have demonstrated its positive effects on cervical and lumbar spine-related pain [4] [5] [6]. However, some review studies [7] [8] [9] pointed out that further research is needed, since there is not enough conclusive evidence to fully support the contribution of the therapy. Therefore, it is important to conduct a qualitative study on the mechanics of the therapy.

\subsection{Dynamic Simulation}

In recent years, along with computer technology advancement, multi-body dynamic simulation engines are now able to realistically simulate real world mechanical systems based on the laws of physics. These advanced physics-based models helped researchers to generate animated simulation of physical events with high degree of precision and flexibility [10] [11]. Physics-based modelling is particularly popular among researchers as it allows the user to interact with the model in real-time with the virtual world and to adjust the various mechanical properties of the model to match the target test subjects. Several studies [11] [12] [13] have developed multi-body cervical spine to study the human head-neck response to impact loading.

\subsection{Purpose of This Research}

The purpose of this research is to better understand the complex cervical spine biomechanics and its behavior during a cervical traction therapy. In order to ve- 
rify the efficacy of cervical traction, it is necessary to first identify the various factors that cause intervertebral separations between the C2 and C7 vertebrae in a cervical traction therapy. While previous studies have examined how different traction angles and forces affect the efficacy [14], it is important to point out that traction position also plays an important role in the efficacy of cervical traction due to the mass distribution of the body. The two most common traction positions are the sitting and supine positions. Previous research studies [15] [16] have evaluated the two positions and concluded that supine position is more effective in terms of the patient's cardiovascular responses and the cervical intervertebral separations. In our research, we conducted a comparative study on the sitting position and the inclined position. In the inclined position, the patient is required to sit on a motorized rotatable chair at an inclined angle. While other research studies focused on the effects of individual patients, our simulation models can allow customizations of body dimension and weights to further examine the effects of the therapy.

In our model, both the human skeleton and the traction equipment are placed together in a physics-based simulation environment, so that we can compare and analyze how different traction angles, forces and traction positions affect the distance of separation between each vertebra. The result will provide insight on the benefits and drawbacks of each traction factors and will serve as a reference for therapists to determine which setup is better under different circumstances. While many physical therapists and patients may not be familiar with the engineering concepts in a cervical traction therapy, a realistic simulation of the cervical vertebrae behavior during the therapy can act as a graphical tool to communicate what actually happens. Furthermore, traction therapy equipment manufacturers can also benefit from this research, as they can use our model to help refine the design of their products.

\section{Methods}

\subsection{Cervical Spine Model}

The cervical spine component was created based on an anatomically correct human skeleton 3D model retrieved from BodyParts3D/Anatomography [17] and it consisted of 8 rigid parts: a human skull and 7 pieces of the cervical vertebrae. In order to minimize processing load in the simulation, all the bones in the skull were combined to create a simple rigid part to represent the skull. All the bones that made up the cranium and the facial area were combined into one rigid body. The cervical vertebrae (C1-C7) were modeled separately and each cervical vertebra was attached to the adjacent vertebrae by a spring-like joint with stiffness and damping settings that mimics the natural behavior of the cervical disc. The lateral flexion and axial rotation were prohibited in the model to simplify the simulation and reduce processing load. Only flexion and extension were allowed in the model.

In previous studies that evaluate the efficacy of traction therapy [12] [14], intervertebral anterior and posterior separations are often used as a reference. By 
defining the reference points along the cervical spine, small sensors were created and attached to the $\mathrm{C} 2$ to $\mathrm{C} 7$ vertebrae. The sensors are weightless so they do not interference with the flexion and extension of the spine. They are attached to the C3, C4, C5, and C6 vertebrae in four positions, namely Anterior Superior, Anterior Inferior, Posterior Superior and Posterior Inferior. In C2, only the inferior side had sensors attached and in C7, only the superior side had sensors attached. The positions of the sensors are shown in Figure 1. Once the sensors were attached to the designated positions of the vertebrae, they moved along with the vertebrae. By simply measuring the distance between sensors, the separation distance between each vertebra could be acquired.

\subsection{Human Skeleton Model}

The human skeleton section of the model included the rest of the body below the cervical spine, and was modelled as separated rigid bodies. Similar to the cervical spine, all the 3D models used in this section were retrieved from BodyParts3D [17]. Each part was imported into 3D modelling software [18] for scaling and re-positioning with the traction equipment. The topmost part of the trunk was cut off at the first vertebra of the lumbar spine (T1) and this would serve as a support for the lowest part of the cervical spine (C7). The two arms were attached to the trunk by ball-socket joints that fixed each arm on to the trunk at a single point and allowed it to move freely in all directions. The legs were separated into upper legs and lower legs. The upper legs (thigh) were attached to the pelvis at the hip and the lower legs (shank) were attached to the upper legs at the knees. All the parts were connected with hinge joints that forced the attached parts to rotate around one axis.

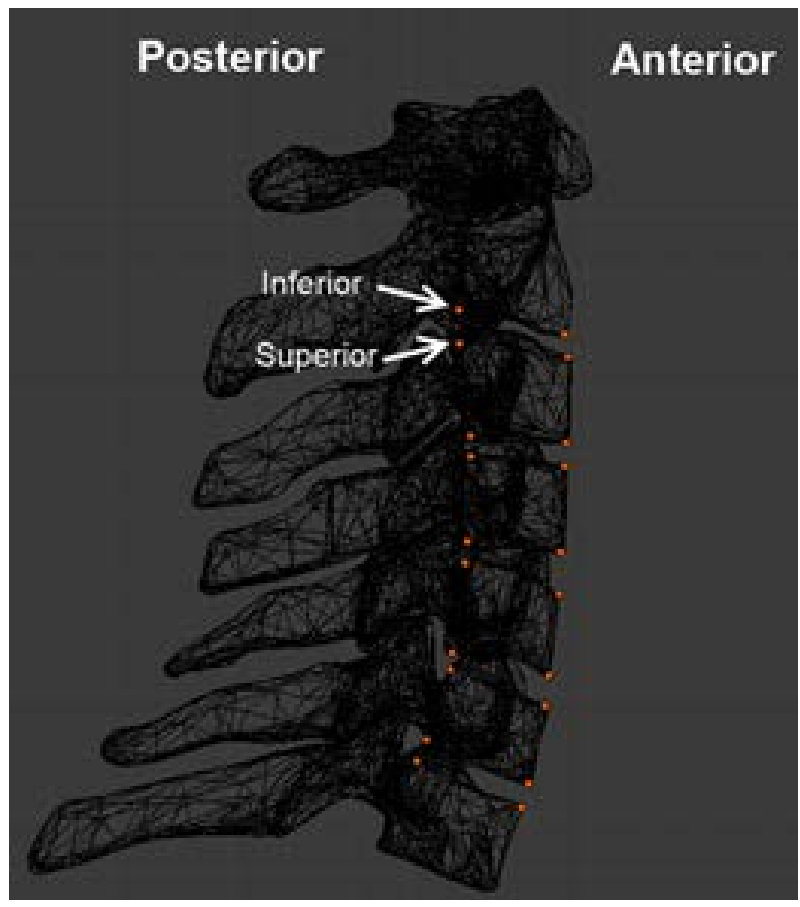

Figure 1. Sensor positions. 
The body segment mass data was configured based on the data from Zatsiorsky et al. with adjustment made by DeLeva [19]. The human body model was $1.74 \mathrm{~m}$ tall and weighted $73 \mathrm{~kg}$. The mass of each bone was calculated based on the percentage listed in the body segment parameter data and is shown in Table 1. The combined head and neck was estimated to be $5.07 \mathrm{~kg}$. It is worth noting this value includes the brain, the cervical spine and all the tissues around the neck.

\subsection{Traction Equipment Development}

A typical set of traction equipment includes a head halter, a pelvic belt, a spreader bar, a rope and a weight. Depending on the design of the head halter, the direction of force varies. Thus, it is important to predefine the points of contact between the head and the halter so to identify the final acting force vector on the head. In the current model, the chin and the back of the head were selected as two contact points for the pulling force to apply on. The top part of the back of the head does not come in contact with the halter when traction is applied during the therapy. It is only when there is no pulling force that the halter will rest on the head. Rendered images of the halter and the points of contact are illustrated in Figure 2.

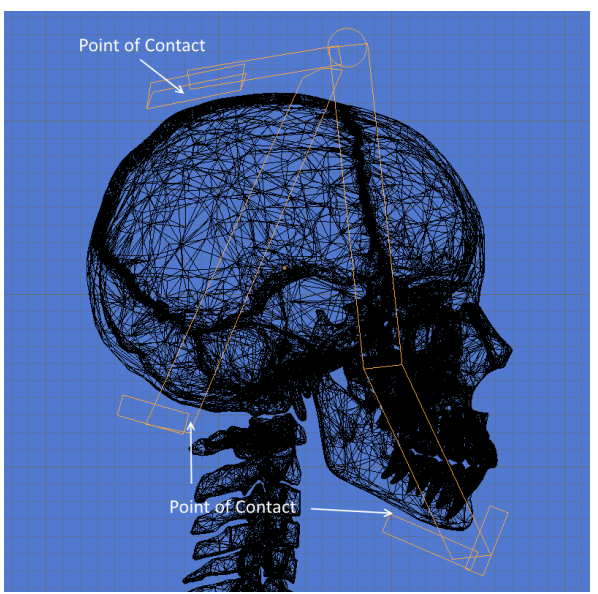

Figure 2. Head halter model.

Table 1. Body Segment Mass

\begin{tabular}{ccc}
\hline Bone Names & Mass (\% of body weight $)^{*}$ & Mass $(\mathrm{kg})$ \\
\hline Head + Neck & 6.94 & 5.07 \\
Upper + Mid Trunk & 32.29 & 23.57 \\
Lower Trunk (pelvis) & 11.17 & 8.15 \\
Upper Arms & $2.71 \times 2$ & $1.98 \times 2$ \\
Forearms + Hands & $2.23 \times 2$ & $1.63 \times 2$ \\
Thighs & $14.16 \times 2$ & $10.34 \times 2$ \\
Shanks + Feet & $5.70 \times 2$ & $4.16 \times 2$ \\
Total & $100 \%$ & 73.0
\end{tabular}


We implemented two types of traction device and they represent the sitting position and the inclined position. In the sitting position, the subject sits up right on a chair with the head attached to a head halter. The traction device applies a constant and continuous force to pull the halter upwards at an angle. The traction force can be set to between $60 \mathrm{~N}$ to $200 \mathrm{~N}$ and the traction angle can be set at $10^{\circ} / 20^{\circ} / 30^{\circ} / 40^{\circ}$. The sitting position was modelled based on the Minato Tractizer TC-30D. The halter is being pulled at $10^{\circ}$ in Figure 3 in the sitting position. In the inclined position, the subject sits on a motorized chair that can be rotated at any angle between $10^{\circ}$ and $40^{\circ}$. The pivot point of the head halter does not change position. The traction angle is controlled only by the rotation of the chair. The inclined position was modelled based on the Minato Tractizer TC-C1. Instead of pulling the subject upward/forward at an angle as in the sitting position, the inclined position rotates the base of the chair to form the traction angle. The name "inclined" describes the position of the rotated chair during the therapy, in which it looks like an incliner chair. This position aims to keep the subject remain in the chair using gravity while at the same time reduce the size of the device. Figure 3 shows the halter being pulled at $40^{\circ}$ in the inclined position. In both models, the traction angle's vertex is at the center point of the subject's first thoracic vertebra (T1). Both simulation models were developed in $\mathrm{C}++$ in Microsoft Visual Studio using Vortex Dynamics 3.0 (CM Labs) [20].

\section{Results}

Simulation runs were performed using combinations of traction angles, forces and positions. In each run, both the anterior and posterior intervertebral separations between $\mathrm{C} 2-\mathrm{C} 7$ vertebrae were measured. Traction angle between $10^{\circ}$ and $40^{\circ}$ and traction force between $60 \mathrm{~N}$ and $200 \mathrm{~N}$ were tested. Both sitting and inclined positions were tested under the same environment, i.e. all the material properties, stiffness and damping parameters of the mechanical joints were the same in both simulations. The measured separations represent the changes of separation before and after traction applied.
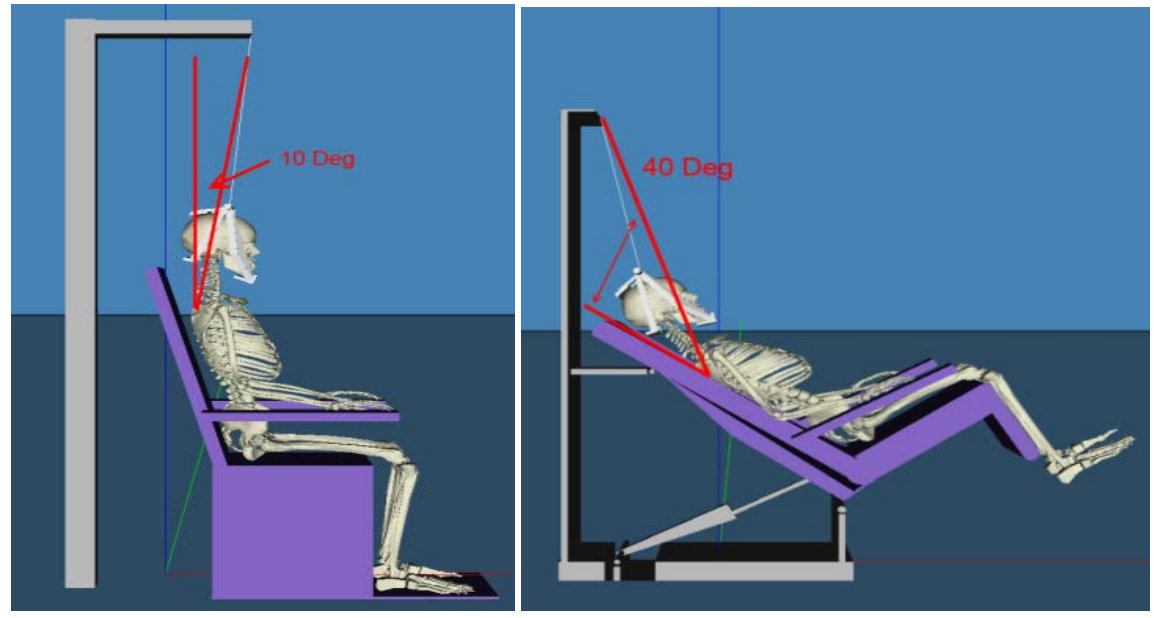

Figure 3. Simulation models in sitting (a) and inclined (b) position. 


\subsection{Intervertebral Separations vs. Time}

The changes of the anterior and posterior separations are shown in Figure 4 and Figure 5. Only the data from $160 \mathrm{~N}$ is presented since the observed behaviors are similar with different traction forces. The measurement represents the overall combined disc space from C2 to C7. On the anterior side in Figure 4, negative separations occurred when traction angles increase. The negative dips were much more noticeable in the sitting position compared to the posterior case. Traction angle at $10^{\circ}$ resulted in the largest separations while $40^{\circ}$ traction angle resulted in compressed cervical spine. Similarly, the posterior side of the sitting position in Figure 5 also shows small dips at the beginning of the test at the $30^{\circ}$ and $40^{\circ}$ lines. The dips become more apparent as the traction angle increases. In the $40^{\circ}$ case, negative separation was observed, indicating that the spine was being compressed rather than extended. Negative separation was not seen in the inclined position. The largest posterior separation was achieved at $10^{\circ}$ and the smallest at $40^{\circ}$. The inclined position led to larger separations in all traction angles when compared to the sitting position. The stretched cervical spine in sitting and inclined positions at $10^{\circ}$ and $40^{\circ}$ are shown in Figure 6 and Figure 7.

\subsection{Individual Intervertebral Separations}

Figure 8 shows the segmental separations, from C2 to C7 on the anterior side.

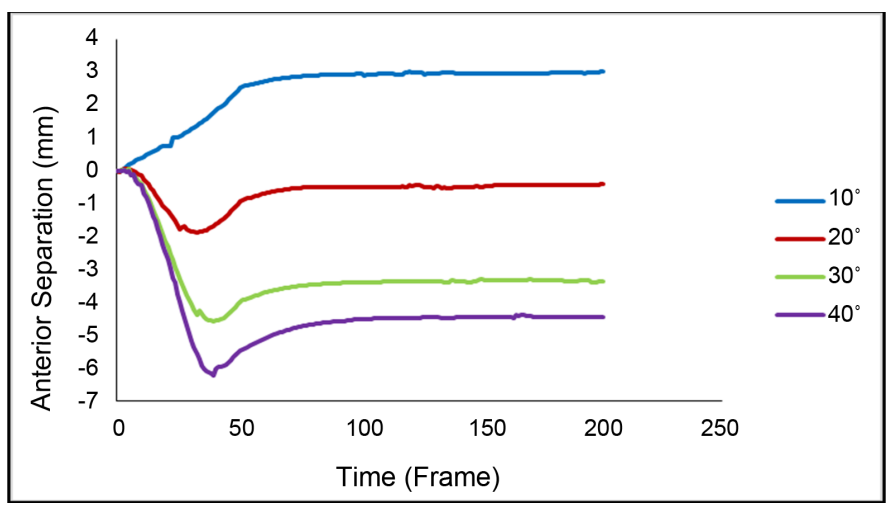

(a)

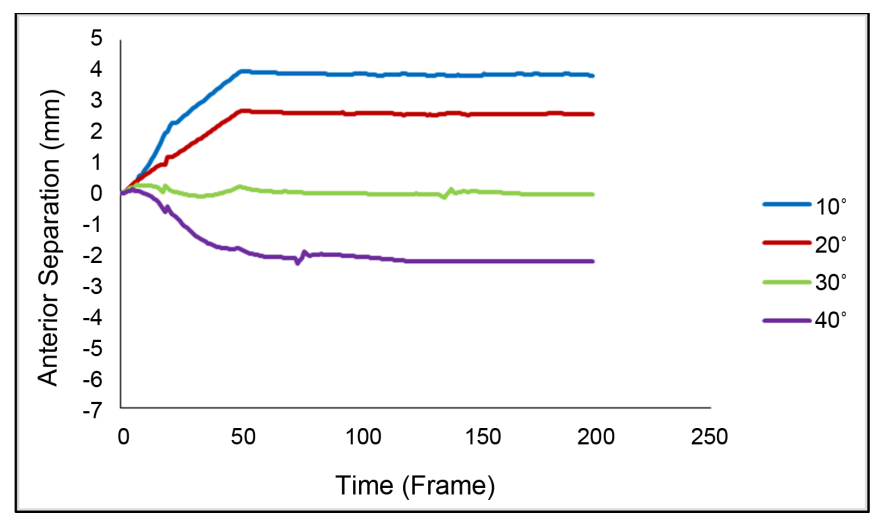

(b)

Figure 4. Anterior Separations at $160 \mathrm{n}$ in sitting (a) and inclined (b) positions. 


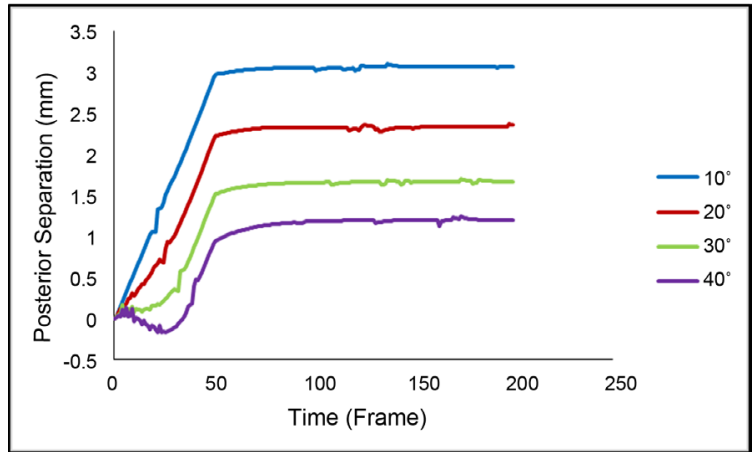

(a)

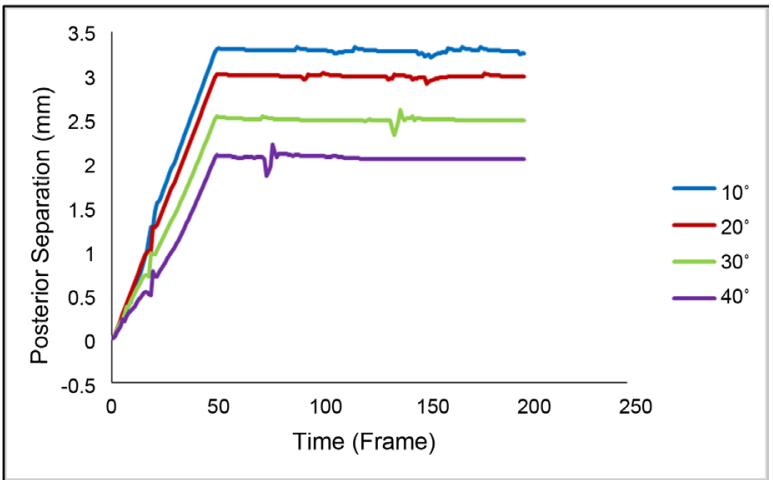

(b)

Figure 5. Posterior Separations at $160 \mathrm{n}$ in sitting (a) and inclined (b) positions.

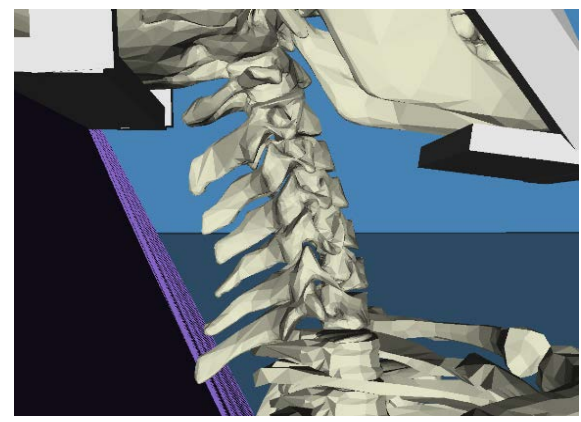

(a)

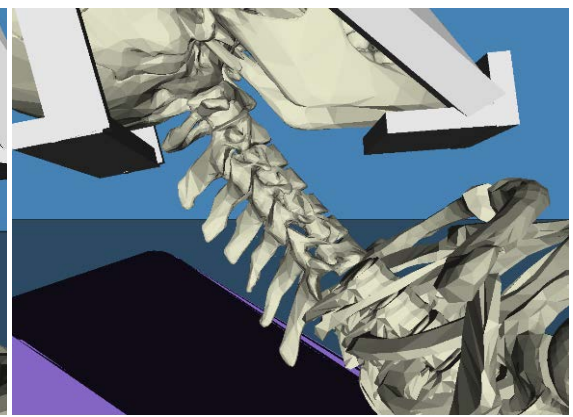

(b)

Figure 6. Sitting position at $10^{\circ}$ (left) and $40^{\circ}$ (right).

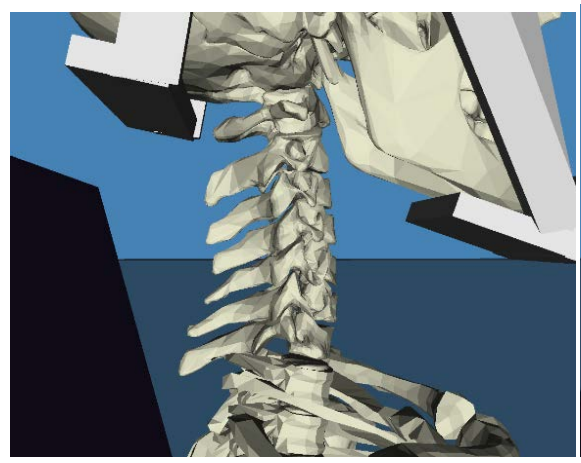

(a)

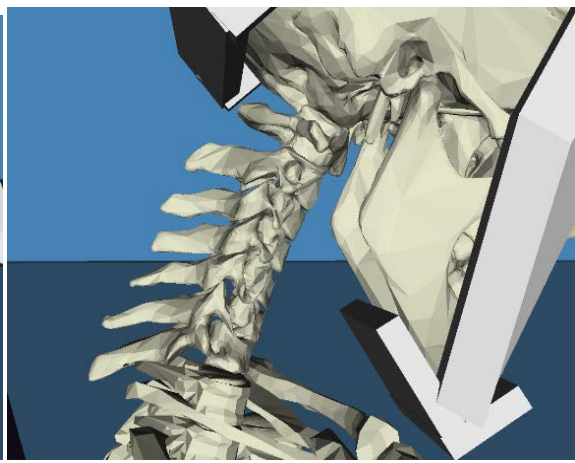

(b)

Figure 7. Inclined position at $10^{\circ}$ (left) and $40^{\circ}$ (right). 


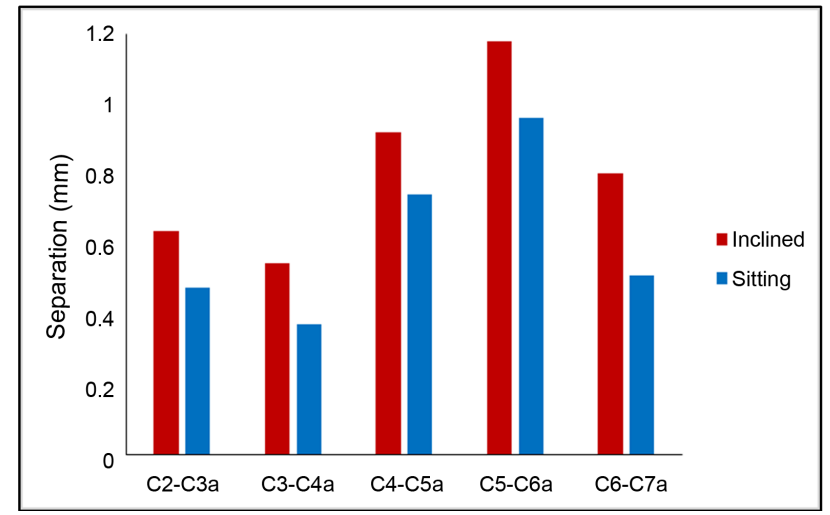

(a)

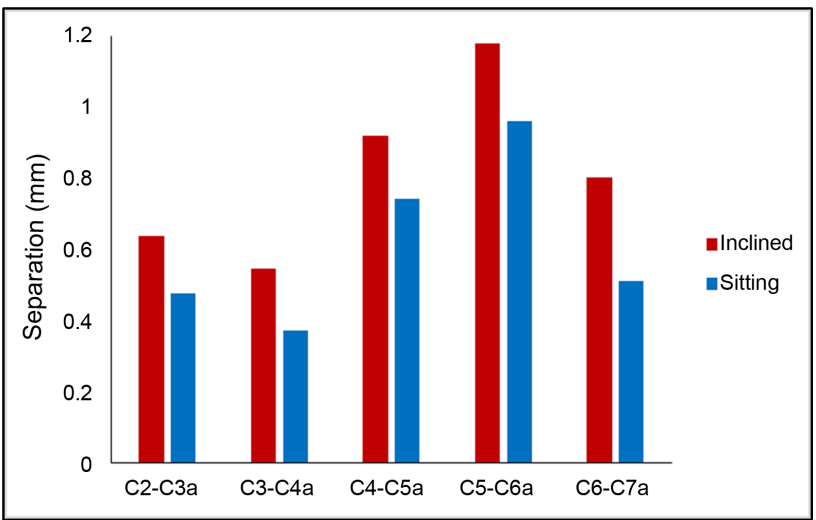

(b)

Figure 8. Anterior intervertebral Separations at $10^{\circ}$ (a) and $40^{\circ}$ (b).

The largest separations were at $10^{\circ}$ and they gradually turned to negative as the traction angle increased. In all cases, the segment C5-C6 was extended the most, followed by the segment $\mathrm{C} 4-\mathrm{C} 5$ and then $\mathrm{C} 6-\mathrm{C} 7$. Overall, the inclined position achieved larger separation and lower compression. The posterior measurements are presented in Figure 9. Again, the segment C5-C6 achieved the highest separation, followed by $\mathrm{C} 4-\mathrm{C} 5$ and $\mathrm{C} 6-\mathrm{C} 7$. The inclined position is found to achieve larger separations than the sitting position. Furthermore, at $40^{\circ}$, the segment C6-C7 in the sitting position achieved the smallest separation.

\subsection{Traction Angles and Traction Forces}

The separations caused by a combination of traction angles and traction forces were compared. Figure 10 shows the anterior separations in the sitting and inclined positions. The negative separation, which indicates that the spine was compressed, can be found in both the sitting and inclined positions. The cervical spine was always compressed further in the sitting position in all combinations of angle and force when compared to the inclined position. Figure 11 shows the posterior separations. On the posterior side, the inclined position was able to achieve a larger separation than the sitting position. When the traction force was small, the sitting position recorded a negative separation in the $30^{\circ}$ and $40^{\circ}$ cases. 


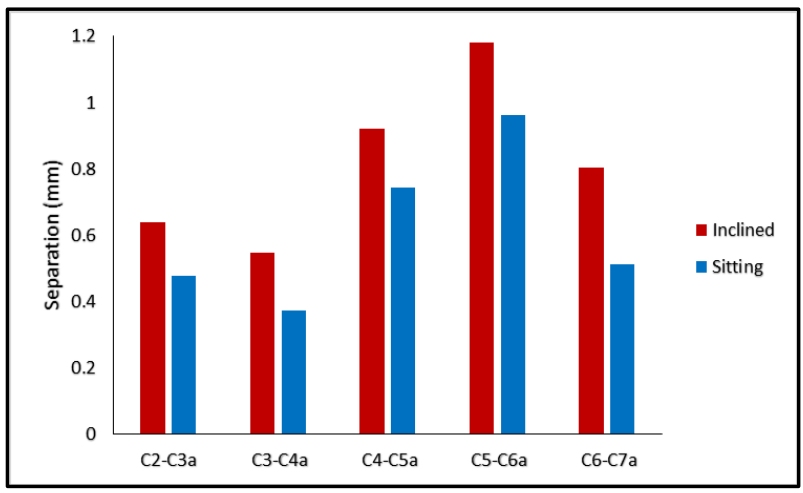

(a)

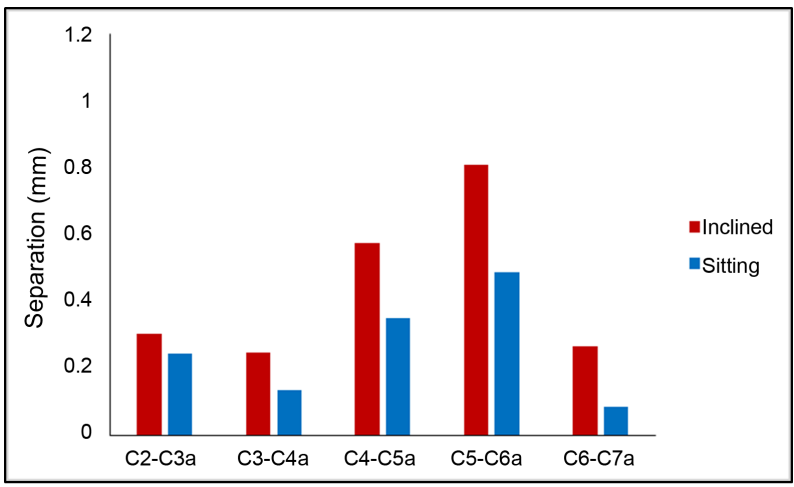

(b)

Figure 9. Posterior intervertebral separations at $10^{\circ}$ (a) and $40^{\circ}$ (b).

\section{Discussion}

The small dips in the sitting position measurements in Figure 4 and Figure 5 are likely related to the forward leaning motion of the body during the traction therapy. In the simulation, we observed that when the subject's head was pulled by the halter, the body stayed in the chair due to the friction force between the lower body and the chair. At this moment, the hip turned and the upper body gradually leaned forward as the traction force increased. The motion stopped when the hip joints reached its limiting angle and could not rotate further. However, the neck continued to bend and created a compression force on the anterior side of the cervical spine. On the other hand, in the inclined position, with the subject resting on a chair at an angle, gravity helped to keep the back of the body remain in contact with the chair. The hip was never turned and the neck did not over bend as in the sitting position. This observation also agreed with a previous study [21], which concluded that anterior separations become negative when traction angle goes beyond $20^{\circ}$. Regarding the segmental separations in Figure 8 and Figure 9, the segment C5-C6 was extended the most, followed by the segment $\mathrm{C} 4-\mathrm{C} 5$ and then $\mathrm{C} 6-\mathrm{C} 7$. These results also agreed with a previous study [21]. The weight of the head and the halter may contribute to the negative separation in the $60 \mathrm{~N}$ case in Figure 10 and Figure 11. In the sitting position, the head and the halter, with a combined weight of $5 \mathrm{~kg}$, exerted a constant downward force to the cervical spine. Since the traction force was at only 


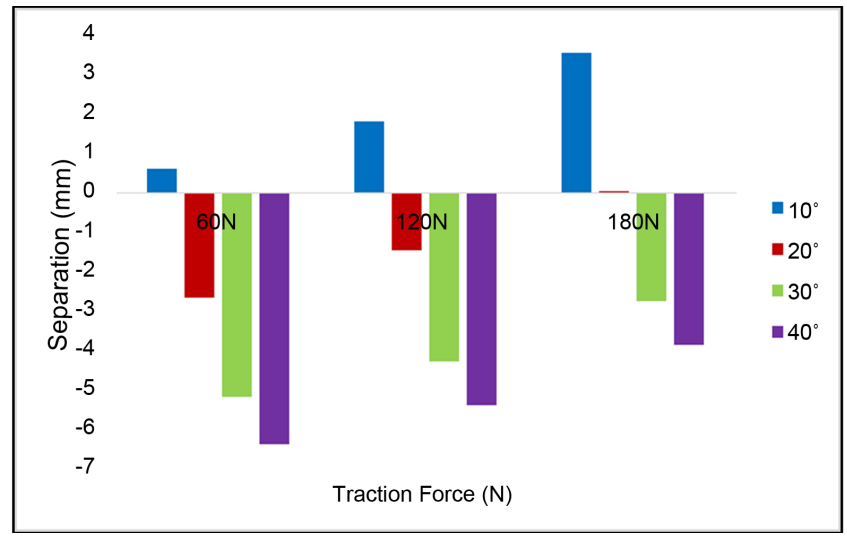

(a)

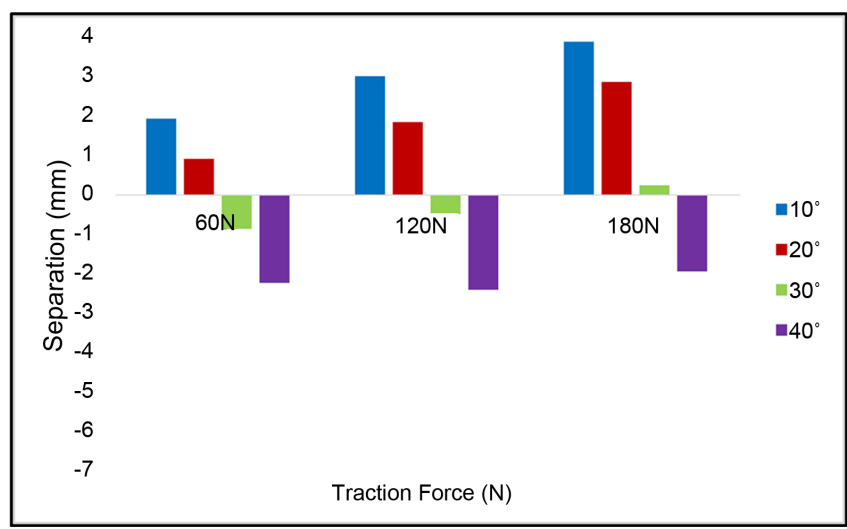

(b)

Figure 10. Traction angle vs Force on anterior in sitting (a) and inclined (b) position.

$60 \mathrm{~N}$, there was very little force to pull the head upward. As the body leaned forward in the sitting position scenario, both the anterior and posterior sides became compressed. Another interesting observation was that the anterior separations reached $-6 \mathrm{~mm}$ in the sitting position at $40^{\circ}$ in Figure 4 , indicating a substantial compression on the anterior side. In contrast, the inclined position only showed a $2 \mathrm{~mm}$ compression under the same condition. On the posterior side, the inclined position was able to achieve a larger separation in all combinations of angles and forces. This may be an indication that the sitting position was adding too much pressure on the anterior side and yet not able to achieve the expected result. Overall, our result suggested that using the inclined position provides a larger separation in both the anterior and posterior sides compared to the sitting position.

Nevertheless, there are several limitations in this model. First of all, the model used a simplified representation of the muscles, ligaments and the intervertebral discs in the cervical spine. As a result, the model does not account for the resistive force from the human subject during the therapy. The model also does not simulate the transient state of a stretched intervertebral dish resulted from traction therapy. Thus, it cannot compare the effect of continuous traction and intermittent traction. 


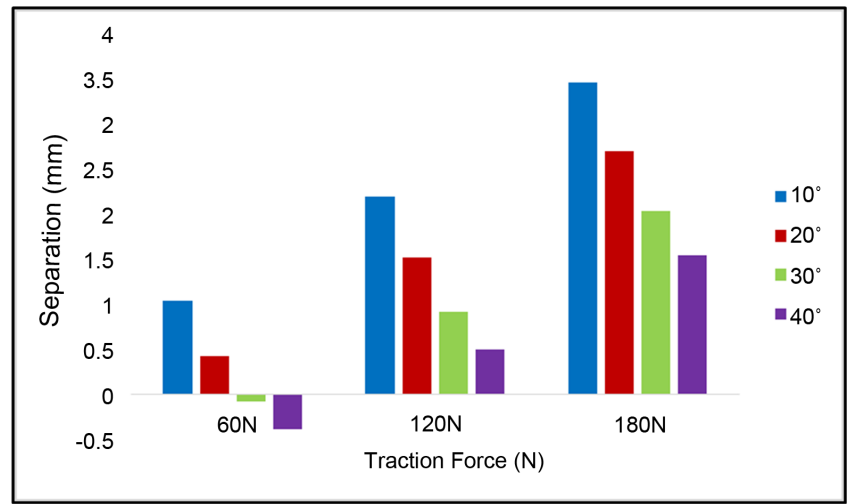

(a)

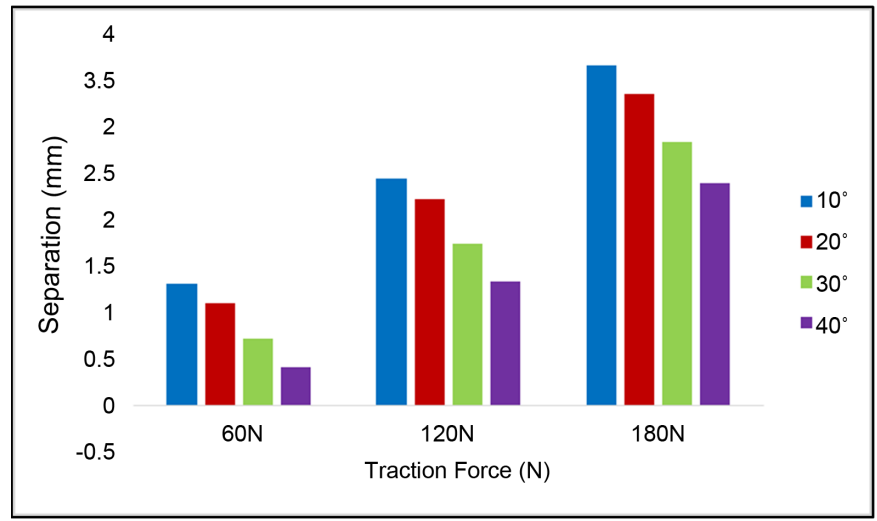

(b)

Figure 11. Traction angle vs force on posterior in sitting (a) and inclined (b) position.

\section{Conclusion}

In order to investigate the effect of traction positions on intervertebral separations of the cervical spine during traction therapy, we constructed a 3D computer simulation model that contains a human skeleton and two traction devices, representing two traction positions. Using this model, the anterior and posterior intervertebral separations were measured in a number of scenarios using various traction forces and angles. The model has several benefits over previous methods. Since the simulation model contains both the subject and the traction devices, the body dimensions of the subject can be easily adjusted to test out different scenarios and new designs of traction device in the future. Also it can serve as a training tool for therapists to evaluate traction positions and demonstrate the effects to patients graphically. In the simulation, we observed that the sitting position could lead to excessive compression at the anterior side at large traction angles and unnecessary forward upper body movement. The inclined position achieved larger intervertebral separations in all combinations of traction forces and angles on both the anterior and posterior sides. This suggests that the inclined position may be more effective in increasing intervertebral separations than the sitting position. The authors would like to extend the research result by performing experimental cervical traction therapies and gather clinical data in the future. 


\section{References}

[1] Haldeman, S., Carroll, L. and Cassidy, J. (2008) The Empowerment of People with Neck Pain: Introduction: The Bone and Joint Decade 20002010 Task Force on Neck Pain and Its Associated Disorders. Spine, 33, S8-S13. https://doi.org/10.1097/brs.0b013e3181643f51

[2] Judovich, B. and Nobel, G.R. (1957) Traction Therapy, a Study Resistance Forces. The American Journal of Surgery, 93, 108-114. https://doi.org/10.1016/0002-9610(57)90748-1

[3] Hogg-Johnson, S., Van Der Velde, G., et al. (2008) The Burden and Determinants of Neck Pain in the General Population: Results of the Bone and Joint Decade 20002010 Task Force on Neck Pain and Its Associated Disorders. European Spine Journal, 33, S39-S51. https://doi.org/10.1097/BRS.0b013e31816454c8

[4] Savva, C. and Giakas, G. (2013) The Effect of Cervical Traction Combined with Neural Mobilization on Pain and Disability in Cervical Radiculopathy. A Case Report. Manual Therapy, 18, 443-446. https://doi.org/10.1016/j.math.2012.06.012

[5] Zylbergold, R. and Piper, M. (1985) Cervical Spine Disorders: A Comparison of Three Types of Traction. Spine, 10, 867-871. https://doi.org/10.1097/00007632-198512000-00001

[6] Jellad, A., Ben Salah, Z., Boudokhane, S., Migaou, H., Bahri, I. and Rejeb, N. (2009) The Value of Intermittent Cervical Traction in Recent Cervical Radiculopathy. Annals of Physical and Rehabilitation Medicine, 52, 638-652.

https://doi.org/10.1016/j.rehab.2009.07.035

[7] van der Heijden, G.J., Beurskens, A.J., Koes, B.W., Assendelft, W.J., de Vet, H.C. and Bouter, L.M. (1995) The Efficacy of Traction for Back and Neck Pain: A Systematic, Blinded Review of Randomized Clinical Trial Methods. Physical Therapy, 75, 93-104. https://doi.org/10.1093/ptj/75.2.93

[8] Harte, A., Baxter, G. and Gracey, J. (2003) The Efficacy of Traction for Back Pain: A Systematic Review of Randomized Controlled Trials. Archives of Physical Medicine and Rehabilitation, 84, 1542-1553. https://doi.org/10.1016/S0003-9993(03)00294-6

[9] Daniel, D.M. (2007) Non-Surgical Spinal Decompression Therapy: Does the Scientific Literature Support Efficacy Claims Made in the Advertising Media? Chiropractic \& Osteopathy, 15, 7. https://doi.org/10.1186/1746-1340-15-7

[10] Coutinho, M.G. (2001) Dynamic Simulations of Multibody Systems. Springer, New York. https://doi.org/10.1007/978-1-4757-3476-8

[11] Merrill, T., Goldsmith, W. and Deng, Y.C. (1984) Three-Dimensional Response of a Lumped Parameter Head-Neck Model Due to Impact and Impulsive Loading. Journal of Biomechanics, 17, 81-95. https://doi.org/10.1016/0021-9290(84)90126-X

[12] Ahn, H. and DiAngelo, D. (2008) A Biomechanical Study of Artificial Cervical Discs Using Computer Simulation. Spine, 33, 883-892. https://doi.org/10.1097/BRS.0b013e31816b1f5c

[13] Wong, L.K., Luo, Z., Kurusu, N. and Fujino, K. (2013) Cervical Spine Simulation Model for Traction Therapy Analysis. Proceedings of the 2013 IEEE/ SICE International Symposium on System Integration, Kobe, 15-17 December 2013, 516-520. https://doi.org/10.1109/SII.2013.6776747

[14] Wong, A., Leong, C. and Chen, C. (1992) The Traction Angle and Cervical Intervertebral Separation. Spine, 17, 136-138. https://doi.org/10.1097/00007632-199202000-00003

[15] Akinbo, S., Danesi, I. and Abudu, M. (2013) Comparison of Supine and Sitting Po- 
sitions Cervical Traction on Cardiovascular Parameters, Pain and Neck Mobility in Patients with Cervical Spondylosis. The Internet Journal of Rheumatology, 8, 1-11. http://ispub.com/IJRH/8/1/1461

[16] Fater, D.C.W. and Kernozek, T.W. (2008) Comparison of Cervical Vertebral Separation in the Supine and Seated Positions Using Home Traction Units. Physiotherapy Theory and Practice, 24, 430-436. https://doi.org/10.1080/09593980802511896

[17] BodyParts3D/Anatomography. Copyright 2008. Database Center for Life Science, Licensed by CC-BY-SA-2.1-jp. http://lifesciencedb.jp/bp3d/?lng=en

[18] Blender 2.75 [Computer Software] (2015). https://www.blender.org/

[19] De Leva, P. (1996) Adjustments to Zatsiorsky-Seluyanov's Segment Inertia Parameters. Journal of Biomechanics, 29, 1223-1230. https://doi.org/10.1016/0021-9290(95)00178-6

[20] Vortex Dynamics 3.0 [Computer software] (2008). http://www.cm-labs.com/

[21] Ito, F. and Kiyama, T. (1985) Angular Factor in Intermittent Cervical Traction [頝 椎間歇牽引における角度因子]. Sogo Rehabilitation [総合リハビリテーション], 13, 213-218.

Submit or recommend next manuscript to SCIRP and we will provide best service for you:

Accepting pre-submission inquiries through Email, Facebook, LinkedIn, Twitter, etc. A wide selection of journals (inclusive of 9 subjects, more than 200 journals) Providing 24-hour high-quality service User-friendly online submission system Fair and swift peer-review system Efficient typesetting and proofreading procedure Display of the result of downloads and visits, as well as the number of cited articles Maximum dissemination of your research work

Submit your manuscript at: http://papersubmission.scirp.org/ Or contact jbise@scirp.org 